\section{Perfil epidemiológico dos portadores de fissuras orofaciais atendidos em um Centro de Referência do Nordeste do Brasil}

\section{Epidemiological characteristics of patients with orofacial clefts attending a Referral Center in Northeast Brazil}

André Luiz Figueiredo Coutinho 1

Marília de Carvalho Lima 2

Marco Antônio Pinto Kitamura 3

José Ferreira Neto 4

Rui Manoel Pereira 5
1,3-5 Centro de Atenção aos Defeitos da Face. Instituto de Medicina Integral Prof. Fernando Figueira - IMIP. Rua dos Coelhos, 300. Recife, PE, Brasil. CEP: 50.070-550.

E-mail: andrecoutinho@hotlink.com.br

2 Pós-Graduação em Saúde da Criança e do Adolescente. Departamento Materno Infantil. Universidade Federal de Pernambuco. Recife, PE Brasil.

\section{Resumo}

Objetivos: descrever a frequência dos tipos de lesão dos portadores de fissura lábio-palatina, verificar a associação entre os fatores demográficos com essas lesões e identificar os fatores associados à idade em que foi realizado o primeiro atendimento médico especializado.

Métodos: este é um estudo do tipo série de casos, com 1216 crianças $\leq 10$ anos portadoras de fissura labial elou palatina não sindrômica atendidas no Centro de Atenção aos Defeitos da Face do Instituto de Medicina Integral Professor Fernando Figueira no periodo de janeiro de 2002 a dezembro de 2005. Os dados foram obtidos de um banco de dados secundário desse Centro.

Resultados: observou-se um discreto predominio do sexo masculino $(57,4 \%)$ e uma maior procedência do Recife/Região Metropolitana. Cerca de metade da amostra $(48,9 \%)$ encontrava-se no grupo etário $\geq 24$ meses quando realizou o primeiro atendimento médico especializado. As crianças cujo primeiro atendimento especializado ocorreu antes dos 12 meses de vida procederam com maior frequência do Agreste e Sertão. Das crianças portadoras de fissura submucosa, 55\% só foram atendidas após os cinco anos de idade.

Conclusões: observou-se que os dados obtidos estão de acordo com os da literatura, no aspecto geral das fissuras. Necessita-se que os profissionais de saúde sejam melhor preparados para atender $e$ diagnosticar as fissuras lábio-palatinas.

Palavras-chave Fenda labial, Fissura palatina, Epidemiologia 
Introdução

As anomalias congênitas afetam cerca de 5\% dos nascidos vivos em todo o mundo e as deformidades craniofaciais constituem um grupo diverso e complexo que incluem anomalias isoladas e múltiplas de etiologia genética ou não. ${ }^{1}$

Entre as más-formações não sindrômicas da região craniofacial as mais comuns são as fissuras labiais com ou sem fissura palatina associada. ${ }^{2,3} \mathrm{As}$ fissuras faciais causam um importante impacto sobre a fala, audição, aparência e cognição, influenciando de maneira prolongada a saúde e a integração social do seu portador, ${ }^{4}$ não só pela morbidade mas, principalmente, pelos distúrbios emocionais, estigmatização e exclusão social, pois interferem no desenvolvimento da autoestima, relações interpessoais e inserção no meio socioeconômico e cultural.4,5 A reabilitação estético-funcional desses pacientes exige atenção multiprofissional integrada e integral, contínua e especializada.

A prevalência das anomalias craniofaciais varia de acordo com a região geográfica e grupo étnico considerado. Dados sobre as anomalias craniofaciais na população brasileira são raros e dispersos, mas, segundo dados do Estudo Colaborativo Latino-americano de Mal-formações Congênitas (ECLAMC), 6 a prevalência no Nordeste para as fissuras lábiopalatinas é de 9,72/10 mil nascidos vivos, e para as fissuras palatinas $2,41 / 10$ mil nascidos vivos. ${ }^{7}$

Pesquisas que traçam o perfil epidemiológico dos pacientes portadores de fissura de lábio e/ou palato, têm identificado fatores associados como: sazonalidade, classe social, etnia, idade dos pais, peso ao nascer, tabagismo, uso de medicamentos e procedência.8-11 Loffredo et al.,12 em investigação pioneira no Brasil, voltada para a realidade do Estado de São Paulo, incluíram além dos aspectos já citados uma análise quantitativa de fatores relacionados à poluição, ingestão de drogas, doenças da mãe, exposição a herbicida/pesticida na lavoura, exposição à radiação ionizante e hereditariedade.

Há carência de estudos na região Nordeste que estabeleçam a prevalência, os fatores de risco e o perfil epidemiológico dos pacientes portadores de fissuras labiais e/ou palatinas. ${ }^{13}$ Como em Pernambuco funciona há nove anos um centro de referência dessa anomalia, este estudo objetivou descrever a frequência dos tipos de lesão dos portadores de fissura lábio-palatina atendidos nesse centro; verificar a associação entre os fatores demográficos com essas lesões e identificar os fatores associados à idade em que foi realizado o primeiro atendimento médico especializado.

\section{Métodos}

Estudo descritivo, realizado no Instituto de Medicina Integral Professor Fernando Figueira (IMIP), localizado na cidade de Recife, Pernambuco, Brasil, onde o Serviço de Cirurgia Plástica, em associação com o Centro de Atenção aos Portadores de Defeitos da Face (CADEFI) do IMIP, oferecem tratamento especializado para os portadores de fendas orofaciais do Nordeste, atuando como o segundo maior centro de referência para essa deformidade no país.

A população foi de 1614 pacientes com idade igual ou menor de dez anos, registrados no banco de dados do CADEFI. Utilizando-se como critérios de exclusão, síndrome genética associada e residir fora do Estado de Pernambuco, foram excluídos 398 casos, obtendo-se uma amostra de 1216 crianças.

Entre janeiro de 2002 e dezembro de 2005 foram atendidos no CADEFI um total de 2408 pacientes e, no ano de 2006 , foram realizados procedimentos cirúrgicos em 473 novos pacientes. Em 2007 foram atendidos 335 casos novos de fissura de lábio e/ou palato em crianças na faixa etária estudada, de um total de 1999 novos pacientes atendidos no ambulatório de pediatria do IMIP.

Como variáveis do estudo foram utilizados os seguintes dados: sexo; idade na primeira consulta; procedência e a classificação diagnóstica quanto ao tipo da fissura: pré-forame (labial), transforame, pré/pós-forame (lábio-palatina) e pós-forame (palatina); extensão da fissura classificada segundo Spina et al.,14 de acordo com o seu posicionamento em relação ao forame incisivo, em completa, incompleta e outras (mistas e submucosas); e a lateralidade da fissura (direita, esquerda ou bilateral).

Informações quanto à história familiar, realização de pré-natal, diagnóstico pré-natal e fatores de risco para fissura labial e/ou palatina estavam ausentes em mais de $75 \%$ das fichas dos pacientes selecionados, o que impossibilitou a análise desses dados.

Os tipos pré/pós-forame e trans-forame foram alocados em um único grupo denominado fissura lábio-palatina; as fissuras pós-forame passaram a ser denominadas fissura palatina e as fissuras préforame, fissura labial. Como o início do tratamento cirúrgico é preconizado aos três meses (fissuras labiais) e nove meses (fissuras palatinas), 15 analisouse separadamente o grupo das crianças que tiveram a primeira consulta com menos de 12 meses de idade.

$O$ banco de dados do serviço, digitado em planilha do Excel, foi exportado para o software EpiInfo, versão 6.04, para realização das análises estatísticas. Utilizou-se o teste do qui-quadrado para 
verificar a associação entre as variáveis categóricas, adotando-se nível de significância de 5\%.

Esta pesquisa foi submetida ao Comitê de Ética em Pesquisa do IMIP, sendo aprovada em Reunião Ordinária sob o número 729.

\section{Resultados}

Verifica-se na Tabela 1 que, dos 1216 pacientes estudados, houve discreto predomínio do sexo masculino $(57,4 \%)$. A maioria das crianças procedeu do Recife na Região Metropolitana (42,8\%). Cerca de metade da amostra $(48,9 \%)$ apresentava idade $\geq 24$ meses na primeira consulta. Quanto ao diagnóstico da fissura, quase a metade da amostra apresentava lesão do tipo lábio-palatina $(48,7 \%)$, a maioria $(66,2 \%)$ era completa em sua extensão e houve predomínio $(49,2 \%)$ da fissura localizada à esquerda.

A Tabela 2 mostra que as fissuras do tipo lábiopalatinas e labiais foram significantemente mais frequentes no sexo masculino, enquanto as fissuras palatinas ocorreram em maior número nas crianças do sexo feminino $(p<0,001)$. Quanto à extensão, verificou-se uma maior ocorrência da fissura completa no sexo masculino $(p=0,07)$; quanto à lateralidade, constatou-se uma maior frequência da fissura bilateral no sexo masculino e as localizadas à esquerda no sexo feminino, porém sem significância estatística $(p=0,12)$. Houve um discreto predomínio da fissura lábio-palatina nas crianças procedentes do Agreste; das fissuras palatinas nas residentes no Recife na Região Metropolitana e das labiais nas do Sertão $(p=0,04)$. Analisando-se a procedência em relação à extensão, verificou-se um menor número de casos de fissuras mistas e/ou submucosas ("outras") oriundas do Agreste (2,7\%), que chegou a ser metade do encontrado no Recife e Região Metropolitana, assim como um maior número de casos de fissuras completas oriundas do Sertão $(p=0,02)$.

Na Tabela 3 verifica-se um maior percentual de crianças com menos de um ano de idade na primeira consulta oriundas do Agreste (46,1\%) e Sertão $(44,6 \%)$, quando comparadas com as do Recife na Região Metropolitana (34,9\%) e Zona da mata $(34,8 \%)(p=0,001)$. Com relação ao tipo da fissura, os portadores de fissura labial chegaram ao Serviço em maior frequência antes do primeiro ano de vida $(45,4 \%)$ em relação aos com fissuras palatinas $(33,2 \%)(p<0,001)$. Quanto à extensão, observou-se que $55 \%$ das fissuras submucosas só receberam atendimento especializado após os cinco anos de idade $(p<0,001)$.
Tabela 1

Características demográficas e dos diagnósticos das fissuras labiais e/ou palatinas de crianças atendidas no CADEFI - IMIP. Recife, janeiro de 2002 a dezembro de 2005.

\begin{tabular}{|c|c|c|}
\hline Variáveis & $\mathbf{N}$ & $\%$ \\
\hline \multicolumn{3}{|l|}{ Sexo } \\
\hline Masculino & 698 & 57,4 \\
\hline Feminino & 518 & 42,6 \\
\hline \multicolumn{3}{|l|}{ Procedência } \\
\hline Recife e Região Metropolitana & 521 & 42,8 \\
\hline Zona da Mata & 210 & 17,3 \\
\hline Agreste & 319 & 26,2 \\
\hline Sertão & 166 & 13,7 \\
\hline \multicolumn{3}{|l|}{ Idade $1^{\text {a }}$ consulta (meses) } \\
\hline $0-11$ & 476 & 39,1 \\
\hline $12-23$ & 145 & 11,9 \\
\hline $24-59$ & 287 & 23,6 \\
\hline $60-120$ & 308 & 25,3 \\
\hline \multicolumn{3}{|l|}{ Tipo da Fissura* } \\
\hline Labio-palatina & 584 & 48,7 \\
\hline Palatina & 331 & 27,6 \\
\hline Labial & 284 & 23,7 \\
\hline \multicolumn{3}{|l|}{ Extensão da Fissura** } \\
\hline Completa & 758 & 66,2 \\
\hline Incompleta & 338 & 29,5 \\
\hline Mista & 29 & 2,5 \\
\hline Submucosa & 20 & 1,7 \\
\hline \multicolumn{3}{|l|}{ Lateralidade da Fissura*** } \\
\hline Esquerda & 406 & 49,2 \\
\hline Bilateral & 216 & 26,2 \\
\hline Direita & 204 & 24,7 \\
\hline
\end{tabular}

*17 casos sem informação quanto ao tipo da fissura; ** 71 casos sem informação quanto à extensão da fissura; *** 390 casos sem informação quanto à lateralidade da fissura.

CADEFI - IMIP = Centro de Atenção aos Portadores de Defeitos da Face do Instituto de Medicina Integral Prof. Fernando Figueira

Observou-se, na Tabela 4, que nas crianças com menos de 12 meses quase metade dos portadores de fissura lábio-palatina $(49,8 \%)$ e um terço dos com fissura palatina $(33,6 \%)$ compareceram à primeira consulta antes do primeiro mês de vida e, naqueles com fissura labial, houve uma maior procura entre dois e três meses de idade $(38 \%)(p<0,001)$. Ao se analisar a extensão da fissura excluíram-se as mistas e submucosas, em virtude do pequeno número de casos. Verificou-se que $43 \%$ das crianças com fissuras completas compareceram ao CADEFI antes de completar um mês de vida $(p=0,02)$. 


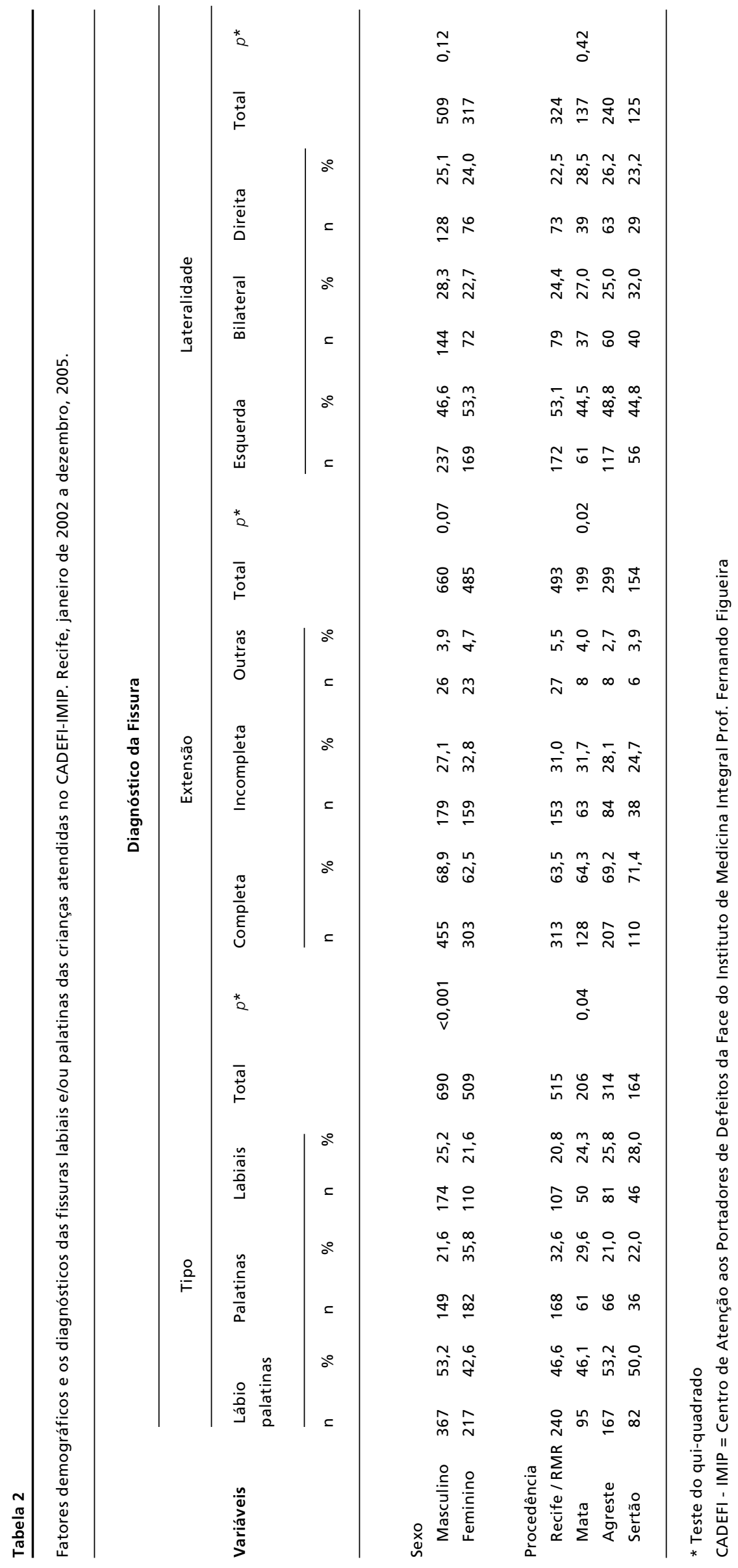


Tabela 3

Fatores demográficos e diagnóstico das fissuras labiais e/ou palatinas de acordo com a idade na $1^{\text {a }}$ consulta médica especializada de crianças atendidas no CADEFI - IMIP. Recife, janeiro 2002 a dezembro 2005.

\begin{tabular}{|c|c|c|c|c|c|c|c|c|}
\hline \multirow{3}{*}{ Variáveis } & \multicolumn{6}{|c|}{ Idade na Primeira consulta (meses) } & \multirow{3}{*}{ Total } & \multirow{3}{*}{$p^{*}$} \\
\hline & \multicolumn{2}{|c|}{$0-11$} & \multicolumn{2}{|c|}{$12-59$} & \multicolumn{2}{|c|}{$60-120$} & & \\
\hline & $\mathrm{n}$ & $\%$ & $\mathrm{n}$ & $\%$ & $\mathrm{n}$ & $\%$ & & \\
\hline \multicolumn{9}{|l|}{ Sexo } \\
\hline Feminino & 197 & 38,0 & 197 & 38,0 & 124 & 24,0 & 518 & \\
\hline Masculino & 279 & 40,0 & 235 & 33,7 & 184 & 26,3 & 698 & 0,28 \\
\hline \multicolumn{9}{|l|}{ Procedência } \\
\hline Recife e Região Metropolitana & 182 & 34,9 & 196 & 37,6 & 143 & 27,5 & 521 & \\
\hline Zona da Mata & 73 & 34,8 & 68 & 32,4 & 69 & 32,8 & 210 & \\
\hline Agreste & 147 & 46,1 & 112 & 35,1 & 60 & 18,8 & 319 & \\
\hline Sertão & 74 & 44,6 & 56 & 33,7 & 36 & 21,7 & 166 & 0,001 \\
\hline \multicolumn{9}{|l|}{ Tipo de Fissura } \\
\hline Lábio-palatina & 229 & 39,2 & 187 & 32,0 & 168 & 28,8 & 584 & \\
\hline Labial & 129 & 45,4 & 90 & 31,7 & 65 & 22,9 & 284 & \\
\hline Palatina & 110 & 33,2 & 148 & 44,7 & 73 & 22,1 & 331 & $<0,001$ \\
\hline \multicolumn{9}{|l|}{ Extensão da Fissura } \\
\hline Completa & 302 & 39,8 & 245 & 32,3 & 211 & 27,9 & 758 & \\
\hline Incompleta & 133 & 39,3 & 143 & 42,3 & 62 & 18,4 & 338 & \\
\hline Mista & 13 & 44,8 & 8 & 27,6 & 8 & 27,6 & 29 & \\
\hline Submucosa & 3 & 15,0 & 6 & 30,0 & 11 & 55,0 & 20 & $<0,001$ \\
\hline \multicolumn{9}{|l|}{ Lateralidade da Fissura } \\
\hline Esquerda & 171 & 42,1 & 132 & 32,5 & 103 & 25,4 & 406 & \\
\hline Bilateral & 81 & 37,5 & 69 & 31,9 & 66 & 30,6 & 216 & \\
\hline Direita & 97 & 47,5 & 56 & 27,5 & 51 & 25,0 & 204 & 0,25 \\
\hline
\end{tabular}

* Teste do qui-quadrado.

CADEFI - IMIP = Centro de Atenção aos Portadores de Defeitos da Face do Instituto de Medicina Integral Prof. Fernando Figueira

\section{Tabela 4}

Diagnóstico das fissuras labiais e/ou palatinas e a idade das crianças menores de 12 meses na $1^{\text {a }}$ consulta médica especializada, atendidas no CADEFI - IMIP. Recife, janeiro 2002 a dezembro 2005.

\begin{tabular}{|c|c|c|c|c|c|c|c|c|c|c|}
\hline \multirow{3}{*}{ Fissuras } & \multicolumn{8}{|c|}{ Idade na Primeira consulta (meses) } & \multirow{3}{*}{ Total } & \multirow{3}{*}{$p^{*}$} \\
\hline & \multicolumn{2}{|c|}{$<1$} & \multicolumn{2}{|c|}{1} & \multicolumn{2}{|c|}{$2-3$} & \multicolumn{2}{|c|}{$4-11$} & & \\
\hline & $\mathrm{n}$ & $\%$ & $\mathrm{n}$ & $\%$ & $\mathrm{n}$ & $\%$ & $\mathrm{n}$ & $\%$ & & \\
\hline \multicolumn{11}{|l|}{ Tipo } \\
\hline Lábio-palatina & 115 & 49,8 & 46 & 19,9 & 50 & 21,6 & 20 & 8,7 & 231 & \\
\hline Labial & 31 & 24,0 & 23 & 17,8 & 49 & 38,0 & 26 & 20,2 & 129 & \\
\hline Palatina & 37 & 33,6 & 22 & 20,0 & 27 & 24,5 & 24 & 21,9 & 110 & $<0,001$ \\
\hline \multicolumn{11}{|l|}{ Extensão } \\
\hline Completa & 130 & 43,0 & 55 & 18,2 & 80 & 26,5 & 37 & 12,3 & 302 & \\
\hline Incompleta & 40 & 30,1 & 28 & 21,1 & 36 & 27,1 & 29 & 21,7 & 133 & 0,02 \\
\hline \multicolumn{11}{|l|}{ Lateralidade } \\
\hline Esquerda & 67 & 39,2 & 29 & 17,0 & 54 & 31,6 & 21 & 12,2 & 171 & \\
\hline Bilateral & 38 & 46,9 & 22 & 27,2 & 13 & 16,0 & 8 & 9,9 & 81 & \\
\hline Direita & 37 & 38,1 & 17 & 17,5 & 26 & 26,8 & 17 & 17,6 & 97 & 0,08 \\
\hline
\end{tabular}

* Teste do qui-quadrado.

CADEFI - IMIP = Centro de Atenção aos Portadores de Defeitos da Face do Instituto de Medicina Integral Prof. Fernando Figueira 


\section{Discussão}

Nesta série de casos observou-se um discreto predomínio de crianças do sexo masculino, estando este percentual de acordo com os da literatura, $6,12,16,17$ cujos dados chegam a uma prevalência de 60 a $80 \%$ dos casos. Com relação ao tipo e extensão da fissura houve, nos casos estudados, predomínio da fissura lábio-palatina completa sobre as demais. Esses achados também são confirmados por Freitas et al.18 e Owens et al., ${ }^{3} \mathrm{em}$ trabalhos distintos.

Com relação à lateralidade, metade das fissuras estavam localizadas à esquerda, seguidas pelas bilateral e direita. A fenda unilateral esquerda é mais frequente que a bilateral, 19 que por sua vez ocorre mais que à direita, chegando-se a afirmar que as fissuras esquerdas podem ser 1,5 vez mais frequentes que as do lado direito. Não há, ainda, uma explicação plausível para esta diferenciação. Acredita-se que, no início da gestação, haja um maior aporte de sangue no lado direito do concepto devido a uma maior pressão sanguínea na carótida interna direita. 18

Ao associar-se o sexo à classificação verificouse, no presente estudo, que as fissuras labial e lábiopalatina foram mais frequentes nos meninos, enquanto a fissura palatina ocorreu mais nas meninas, confirmando os relatos da literatura. 2,11,17,19-21

Quando a procedência foi o foco da investigação, obteve-se um significante predomínio da fissura palatina nas crianças residentes no Recife e Região Metropolitana. Embora os dados desta pesquisa não nos permitam realizar tal inferência, em geral, nos grandes centros urbanos, observa-se uma maior exposição aos agentes poluentes ambientais, o que poderia ter influenciado a ocorrência desse achado, o qual já foi descrito por Loffredo et al. ${ }^{12}$ Por outro lado, as fissuras labiais mais relacionadas com fatores hereditários, foram mais preponderantes nas crianças provenientes do Agreste e Sertão, provavelmente secundária a uma maior consanguinidade observada em populações residentes no interior. No entanto, essas conjecturas necessitam ser exploradas em estudos futuros utilizando desenhos epidemiológicos adequados.

As diferenças observadas quanto ao tipo da lesão podem ser devidas a uma possível influência de fatores sociodemográficos inerentes a cada região. Pesquisa realizada no estado do Missouri, Estados Unidos22 demonstrou diferença estatisticamente significante entre neonatos, portadores de fissuras faciais, advindos das zonas urbana e rural.
Observou-se que a idade do paciente na primeira consulta médica especializada não esteve associada ao sexo nem à lateralidade da fissura; no entanto, houve associação significante entre esta e a procedência, tipo e extensão da lesão. Assim sendo, ao contrário do esperado, a primeira consulta foi realizada mais frequentemente durante o primeiro ano de vida nas crianças provenientes do Sertão e Agreste. Esse achado pode ser justificado pelo fato dos municípios localizados a mais de $50 \mathrm{~km} \mathrm{da}$ capital fazerem jus a um benefício do governo federal: o tratamento fora de domicílio (TFD), em que os gastos com o transporte destes pacientes são repassados para as prefeituras. Paradoxalmente, esta situação fez com que houvesse retardo na chegada das crianças residentes na Zona da Mata e Região Metropolitana, fazendo com que houvesse predomínio de crianças dessas áreas com idade acima de cinco anos. Esse dado é relevante porque mostra que crianças residentes na capital e Região Metropolitana têm acesso ao tratamento de uma forma tardia e que isso implica em prejuízo ao sucesso do mesmo. Desta forma é importante a realização de campanhas de conscientização dos profissionais de saúde em referenciar esses pacientes em tempo hábil para dar início ao tratamento especializado.

Os portadores de fissuras labial e lábio-palatina tiveram maior frequência do primeiro atendimento médico especializado antes dos 12 meses de vida; provavelmente por serem essas lesões mais evidentes, houve preocupação dos pais em procurar atendimento especializado mais rapidamente nesse grupo, enquanto nas fissuras palatinas, por não serem aparentes, a idade do primeiro atendimento ocorreu mais frequentemente após o primeiro ano de idade.

Quanto à extensão da fissura, observou-se um dado alarmante, pois metade das crianças com fissuras submucosas só chegaram ao serviço após os cinco anos de idade, contribuindo para o grave comprometimento da recuperação da fonação desses pacientes. A falta de treinamento das equipes de saúde associada à dificuldade de diagnóstico precoce justificam esse retardo.

O grupo de crianças atendidas com menos de 12 meses de idade na primeira consulta foi então analisado separadamente para verificar se estariam chegando em tempo hábil para o início do tratamento cirúrgico. Observou-se que o tipo da fissura teve uma significante influência na busca de tratamento especializado. Cerca de metade das crianças com fissuras lábio-palatinas compareceram antes do primeiro mês de vida, enquanto apenas $8,7 \%$ foram 
atendidos após o quarto mês. Assim sendo, para esse grupo de crianças, quase a totalidade dos portadores desse tipo de fissura realizaram a cirurgia corretiva na idade preconizada.

Devido ao elevado percentual de gestantes iniciarem o pré-natal após o primeiro trimestre da gestação, foi dado um passo importante pelas autoridades sanitárias, através de consulta pública da Agencia Nacional de Vigilância Sanitária (ANVISA), 23 que instituiu o acréscimo de ácido fólico em todos os farináceos utilizados na indústria alimentícia. Essa medida, com certeza, ocasionará uma redução nas taxas de malformações do tubo neural e craniofacial, sabidamente decorrentes de deficiência desse ácido nos primórdios da gestação. 24

\section{Referências}

1. Monlleó IL, Lopes VLG. Anomalias crânio-faciais: descrição e avaliação das características gerais de atenção no Sistema Único de Saúde. Cad Saúde Pública. 2006; 22: 913-22.

2. Loffredo LCM, Freitas JAS, Grigolli AAG. Prevalência de fissuras orais de 1975 a 1994, Brasil. Rev Saude Publica. 2001; 35: 571-5.

3. Owens JR, Jones JW, Harris F. Epidemiology of facial clefting. Arc Dis Child. 1985; 60: 521-4.

4. Amaral VLAR. Aspectos psicológicos, problemas sociais e familiares associados às fissuras lábio-palatinas. In: Carreirão S, Lessa S, Zanini AS, editores. Tratamento das fissuras labiopalatinas. Rio de Janeiro: Revinter; 1996. p. 19-23.

5. Chan RK, McPherson B, Whitehill TL. Chinese attitudes toward cleft lip and palate: effects or personal contact. Cleft Palate Craniofac J. 2006; 43: 731-9.

6. Nazer J, Hubner ME, Catalán M. Incidência de lábio leporino y paladar hendido en la Maternidad Del Holpital Clínico de la Universidad de Chile y en las maternidades chilenas participantes en el Estúdio Colaborativo Latino Americano de Malformaciones Congênitas (ECLAMC). Rev Méd Chile. 2001; 129: 285-93.

7. Castilla EE, Lopez-Camelo JS, Paz JE. Atlas geográfico de las malformaciones congênitas en Sudamérica. Rio de Janeiro: Fiocruz; 1995.

8. Fraser FC, Gwyn A. Seasonal variation in birth date of children with cleft lip. Teratology. 1998; 57: 93-5.

9. Rintala A, Pönka A, Sarna S, Stegars T. Cleft lip and palate in Finland in 1948-1975: correlations to infections, seasonal and yearly variations. Scand J Plasr Reconstr Surg. 1983; 17: 197-201.

10. Rajesh P, Rajesh R, Narayanan V, Baig MF, Prabhu VR, Venkatesan A. A clinical profile to assess the potencial risk factors for cleft lip and palate. J Indian Soc Pedod Prev Dent. 2000; 18: 147-50.
Os dados obtidos no presente estudo estão de acordo com os da literatura no que diz respeito aos aspectos gerais das fissuras. Entretanto, obtivemos dados bastante peculiares à realidade do nosso Estado, principalmente nas variações entre as microrregiões. Como foi um estudo pioneiro, houve dificuldade na comparação de experiências de outros serviços da região. Através desta pesquisa constatouse a necessidade de padronizar o formulário de atendimento do Centro, instituindo-se uma nova ficha de avaliação com dados sociodemográficos dos pais e, principalmente, a inclusão padronizada dos fatores de risco associados às fissuras, o que ampliará, sobremaneira, a qualidade dos dados necessários para a realização de futuros trabalhos sobre o tema.

11. Toranzo Fernández JM, Metlich Medlich MA, Santos Diaz MA, Vega Estrada NL. Fissura lábio-palatina: análisis epidemiológico de 121 pacientes. Rev ADM. 1993, 50: 165-7.

12. Loffredo LCM, Souza JMP, Yunes J, Freitas JAS, Spiri WC. Fissuras labiais: estudo caso-controle. Rev Saúde Pública. 1994; 28: 213-7.

13. Varandas ET, Silva EC. Fissuras lábio-palatinas: análise epidemiológica no hospital universitário Lauro Wanderley. Ciência, Cultura, Saúde. 1995; 14: 94-103.

14. Spina V, Psillakis JM, Lapa FS, Ferreira MC. Classificação das fissuras lábiopalatinas. Sugestão de modificação. Rev Hosp Clin Fac Med São Paulo. 1972; 27: 5-6.

15. Martins DMFS. Fissuras labiais e palatinas. In: Ferreira LM, editora. Manual de cirurgia plástica. São Paulo: Atheneu; 1995. p. 165-73.

16. Cunha ECM, Fontana R, Fontana T, Silva WR, Moreira QVP, Garcias GL, Roth MGM. Antropometria e fatores de risco em recém-nascidos com fendas faciais. Rev Bras Epidemiol. 2004; 7: 417-22.

17. Palomino H, Guzmán E, Blanco R. Recurrencia familiar de labio leporino con o sin fisura velopalatina de origem no sindrómico en poblaciones de Chile. Rev Méd Chile. 2000; 128: 286-93.

18. Freitas JAS, Dalben GS, Santamaria Junior M, Freitas PZ. Informações atuais sobre a caracterização das fissuras orofaciais no Brasil. Braz. Oral Res. 2004; 18: 128-33.

19. Magdalenic-Mestrovic M, Bagatin M. An epidemiological study of orofacial clefts in Croatia 1988-1998. J Craniomaxillofac Surg. 2005; 33: 85-90.

20. Carinci F, Rullo R, Farina A, Morano D, Festa VM, Mazzarella N, Del Viscovo D, Carls PF, Becchetti A, Gombos F. Non-syndromic orofacial clefts in Southern Italy: pattern analysis according to gender, history or maternal smoking, folic acid intake and familial diabetes. J Craniomaxillofac Surg. 2005; 33: 91-4. 
21. Meng T, Shi B, Zheng Q, Wang Y, Li S. Clinical and epidemiologic studies of nonsyndromic cleft lip and palate in China: analysis of 4268 cases. Ann Plast Surg. 2006; 57 : 264-9.

22. Missouri. Department of Health. Center for Health Information Mangament \& Epidemiology. Provisional statistics. Missouri Monthly Vital Stat. [serial online]. 2000; 34(1). [consulted on: 2006 Jun 6]. Available from: URL: http://www.dhss.missouri.gov/FOCUS/Mar2000.pdf
23. ANVISA (Agência Nacional de Vigilância Sanitári). A farinha terá ácido fólico para combater anencefalia em bebês. Informes Técnicos, 2002. [serial online]. [acesso em: 2006 jul.6]. Disponível em: http://www.anvisa.gov.br/ divulga/informes/2002/120602.htm

24. Nasser C, Nobre C, Mesquita S, Ruiz JG, Carlos HR, Prouvot L, Yacubian MT. Semana da conscientização sobre a importância do ácido fólico. J Epilepsy Clin Neurophysiol. 2005; 11: 199-203.

Recebido em 9 de novembro de 2007

Versão final apresentada em 17 de fevereiro de 2009

Aprovado em 18 de março de 2009 